\title{
Accommodating pregnant emergency physicians
}

\author{
Lisa A. Calder, $\mathrm{MD}^{*}$; A. Adam Cwinn, MD*
}

Pregnant emergency physicians have unique needs that may not be explicitly addressed by departmental policy or culture within emergency departments (EDs). Essentially, the issue is how to optimize the health and safety of pregnant emergency physicians and their offspring. The policy Accommodating Pregnant Emergency Physicians: A Guiding Document, which our department developed and implemented (Appendix), and its rationale and methodology are explained.

The changing physician workforce in Canada has recently garnered media attention. ${ }^{1,2}$ Emergency medicine is impacted given that the supply of physicians is dynamic over time, changing not only in the age demographic but also most notably in the proportion of female physicians. ${ }^{3,4}$ This is implied from national data from physician workforce statistics as there are no data for emergency medicine. If this assumption holds, it is fair to say that women are becoming a significant proportion of the emergency physician workforce.

There is no literature on the risk to pregnant women or the fetus during work in an ED. Existing evidence about shift workers indicates an association between shift work, particularly night shifts, and preterm births and small for gestational age infants., ${ }^{5,6}$ This is particularly true in the second and third trimesters. The literature on the impact of residency training on pregnancy also suggests an increased risk of pregnancy complications. ${ }^{7}$ Arising out of this limited literature, the Physician Health Program of British Columbia took the lead in 2010 and published Medicine and Motherhood: Can We Talk? ${ }^{2}$ This consensus document summarized current literature and made general recommendations to facilitate the discussion between pregnant physicians, their colleagues, and their employers. This report was discussed at the Canadian Association of Emergency Physicians (CAEP) Women in Emergency Medicine Interest Group at the 2010 CAEP conference in Montreal.

Following on this discussion, we reflected on our local experience at The Ottawa Hospital. Women who had been pregnant in the early 1980s indicated that they had since noticed a significant improvement in the physician culture with respect to accommodating pregnant emergency physicians. Looking ahead at the demographics of our group, we could anticipate that many more pregnancies were forthcoming. Our current leadership cultured a positive environment, such that women felt comfortable disclosing pregnancy early, and it was understood that shift accommodations would be made near-term, but there was no policy or formal structure. As a result, we struck a working group to address the needs of pregnant emergency physicians. This working group reviewed the literature on shift work and pregnancy, including the Physician Health Program of British Columbia document. ${ }^{8} \mathrm{We}$ undertook broad consultation with the other members of our department, including an online survey of members regarding their previous pregnancy experiences. This process illuminated multiple factors and resulted in recommendations spanning the prepregnancy, pregnancy, and postpartum periods (see the Appendix). The major principles underlying these recommendations are outlined below:

1. Prepregnancy. We found that female emergency physicians needed a general awareness of an existing departmental guide that would allow for accommodation during pregnancy. This would

From the *Department of Emergency Medicine, University of Ottawa, and †Clinical Epidemiology Program, Ottawa Hospital Research Institute, Ottawa, ON.

Correspondence to: Dr. Lisa A. Calder, The Ottawa Hospital - Civic Campus, 1053 Carling Avenue, F658, Box 685, Ottawa, ON K1Y 4E9; Icalder@ ohri.ca.

This article has been peer reviewed.

(C) Canadian Association of Emergency Physicians 
alleviate much anxiety about practice concerns while pregnant.

2. Pregnancy. Early disclosure of pregnancy: A supportive environment was emphasized to allow for a confidential early disclosure to occur with the department head. The timing of this disclosure was to be left to the woman's discretion, either 12 weeks' gestation or earlier. This would allow for human resource planning and shift scheduling for both the departmental needs and the needs of the pregnant emergency physician.

Graduated reduction in physically taxing shifts near term: A number of physical challenges exist when competently performing critical procedures in the third trimester (e.g., intubation). Based on a case control study by Croteau and colleagues, we suggested stopping resuscitation shifts at 28 weeks' gestation and stopping night shifts at 24 weeks' gestation. ${ }^{6}$ This study demonstrated a reduction in the risk of small for gestational age infants when irregular shift work, lifting heavy loads, and highly psychologically demanding work were eliminated at 24 weeks of pregnancy. We also recommended that shift volume be reduced by $25 \%$ during the third trimester and that shifts be limited to daytime and ambulatory care shifts in the last 4 weeks of clinical work.

Working conditions: Emergency medicine comes with attendant risks of exposure to infectious agents, which may not be known at the time of presentation. We recommended that pregnant emergency physicians consider requesting their serum titres of immunity to varicella and parvovirus B19. Although not a guarantee of protection, this may assuage unforeseen postexposure concerns. When possible, it would be ideal for another emergency physician on duty at the time to evaluate the very ill febrile patient or the undifferentiated rash.

3. Maternity leave. It was acknowledged that, historically, many female physicians have taken very brief maternity leaves (2-4 months). ${ }^{9}$ We highlighted awareness of provincial medical association parental leave benefits (ranging from 15 weeks in British Columbia, to 17 weeks in Ontario, to 20 weeks in Saskatchewan). ${ }^{10-12}$

No specific duration of maternity leave was recommended, although it was noted that it was variable within our group but growing longer. In most cases, it was not the 52 weeks that is the national norm. ${ }^{13}$
4. Returning to work in the postnatal period. Graduated increase in shifts: We found that starting a return to work with a night shift or a resuscitation shift was not ideal. Some returning emergency physicians may wish to initially work part-time until child care arrangements are well established. The first 2 weeks of return to work should not include night shifts and should begin in ambulatory care (or urgent care). We suggested that returning emergency physicians consider undertaking a practical clinical procedural skills refresher (either via a simulation centre or a continuing medical education course). A private space close to the ED was made available for mothers who wished to pump breast milk while on shift.

The issue of how to accommodate pregnant emergency physicians is of national relevance and increasingly will become an active issue for all EDs. We encourage Canadian EDs to engage in an open dialogue on what their departmental needs are and consider policy development in this area. The time is right for dialogue on this issue at a national level. A national survey of Canadian emergency physicians on the topic of accommodating women in pregnancy and physicians who are parents is complete and analysis is under way (Dr. Jennifer Williams, personal communication, 2012). Once data are available on emergency physicians' perceived needs around this issue, we would urge the CAEP to consider a position statement to help Canadian EDs move forward.

Acknowledgements: Thank you to the working group members who helped develop The Ottawa Hospital Department of Emergency Medicine guiding document for accommodating pregnant emergency physicians: Drs. Justine Amaro, Jennifer Clow, Soraya Davoudi, Gary Greenberg, Elisabeth Hobden, Christine Johns, Barb Miller, Janet Nuth, Jacky Parker, Shena Riff, Kari Sampsel, and Alena Spacek.

Competing interests: None declared.

\section{REFERENCES}

1. Globe and Mail. Physician turnover rates mirror economic conditions and bousing market. accessed January 23, 2013.

2. CBC News. Doctor numbers in Canada at all-time bigh. Available at: www.cbc.ca/news/health/story/2011/12/15/ doctos-canada-report.html (accessed January 23, 2013).

3. Tyrell L, Dauphinee D. Task force on physician supply in Canada. Canadian Medical Association; 1999.

4. Weizblit N, Noble J, Baerlocher MO. The feminisation of Canadian medicine and its impact upon doctor productivity. 
Med Educ 2009;43:442-8, doi:10.1111/j.1365-2923.2009. 03309.x.

5. Mozurkewich EL, Luke B, Avni M, et al. Working conditions and adverse pregnancy outcome: a meta-analysis. Obstet Gynecol 2000;95:623-35, doi:10.1016/S0029-7844(99) 00598-0.

6. Croteau A, Marcoux S, Brisson C. Work activity in pregnancy, preventive measures, and the risk of delivering a small-for-gestational-age infant. Am 7 Public Health 2006; 96:846-55, doi:10.2105/AJPH.2004.058552.

7. Finch SJ. Pregnancy during residency: a literature review. Acad Med 2003;78:418-28, doi:10.1097/00001888-20030400000021.
8. Physician Health Program of British Columbia. Medicine and motherhood: can we talk? A consensus statement. March 2010.

9. Lent B, Phillips S, Richardson B, Stewart D. Promoting parental leave for female and male physicians. CMAJ 2000; 162:1575-6.

10. Paul K. BCMA insurance editor page. Available at: https:// www.bcma.org (accessed March 20, 2013).

11. Kostiuk J. Parental leave fund. Available at: https://www.sma. sk.ca (accessed March 20, 2013).

12. Ontario Medical Association. Pregnancy and parental leave benefit program. Available at: https://www.oma.org (accessed March 20, 2013).

13. Canadian Labour Code, R.S.C. 1985, c. L-2 (section 206.2). 